\title{
Visual evoked potential correlates of early neural filtering during selective attention
}

\author{
ROBERT G. EASON \\ University of North Carolina, Greensboro, North Carolina 27412
}

\begin{abstract}
Using controls and procedures responsive to criticisms of an earlier study by Eason, Harter, and White (1969), two experiments were conducted to test the hypothesis that selectively attending to a given spatial location involves a corticofugally controlled precortical neural filter that differentially screens information arriving from relevant and irrelevant locations. Twelve subjects participated in each experiment. The amplitude of an early evoked potential component believed to arise from striate cortex was greater to attended than to unattended stimuli presented in a given visual field. The amplitude also was greater during focused than during divided attention. In addition to corroborating the 1969 study, the results supported the precortical filtering hypothesis.
\end{abstract}

The primary purpose of this study was to ascertain whether relevant (i.e., attended) information exerts a greater influence on primary visual cortex than does irrelevant (unattended) information due to the activation of a centrifugal feedback system that modulates visual activity precortically. Several lines of evidence support such a hypothesis, including observations that visual evoked potential (VEP) components with latencies of $100 \mathrm{msec}$ or less are affected by stimulus relevancy (Eason, Harter, \& White, 1969; Van Voorhis \& Hillyard, 1977), that centrifugal fibers project to all levels of the visual system (Hasselt, 1973; Livingston, 1978), and that sensory transmission is influenced by changes in an animal's attentional state (Oatman \& Anderson, 1980). No clear evidence, however, has been provided by human VEP studies to date supporting the existence of such a filtering mechanism (Hillyard, Picton, \& Regan, 1978).

Since the earliest human VEP component resembles that recorded from the striate cortex of animals under near-threshold conditions (Creutzfeldt \& Kuhnt, 1973), it seems probable that this component $(60-$ to $70-\mathrm{msec}$ onset latency) reflects such activity in humans. This study, consisting of two experiments, attempts to test the precortical filtering hypothesis by demonstrating that the early VEP component can be amplitude modulated by variations in selective attention. In addition, this study replicates the 1969 study by Eason et al., using procedures and controls responsive to criticisms by Näätänen (1975) and others.

The first experiment reported herein was presented at the 1976 meeting of the Psychonomic Society in St. Louis; the second was presented at the 1977 meeting of the Eastern Psychological Association in Boston. The author expresses his appreciation to George Ritchie for his participation in the collection and analysis of the data.

\section{Experiment 1}

As in the Eason et al. (1969) study, a paradigm was employed wherein subjects responded to stimulus flashes presented in one visual field (relevant field) while they attempted to ignore and not respond to flashes presented at the homologous site in the opposite (irrelevant) field. Stimuli were presented at two new locations (10 deg and $30 \mathrm{deg}$ ) along the horizontal meridian to establish whether selective attention effects become more pronounced as the relevant and irrelevant stimuli are spaced farther apart. The interaction effects of selective attention with the visual field in which the evoking stimulus is presented, the lobe from which occipital potentials are recorded, and the directness of information transmission from the hemiretina stimulated to the lobe recorded from (primary vs. secondary) also were studied.

\section{Experiment 2}

The paradigm used in Experiment 1 and the earlier study was expanded to study the influence of divided attention on the early VEP component. As in the Eason et al. (1969) study, stimuli were presented at 20-deg eccentricity. Subjects were required to (1) respond to flashes appearing in a given visual field while attempting to ignore and not respond to flashes appearing in the opposite field, (2) respond to stimuli appearing in both fields, and (3) respond to brightness variations in the fixation (foveal) stimulus while "ignoring" flashes appearing peripherally in each field. These manipulations resulted in (1) one field's being relevant (focused attention), (2) one's being irrelevant, (3) both being relevant (divided attention), and (4) both being irrelevant.

\section{METHOD}

\section{Design}

In Experiment 1 the design consisted of five factors with two levels each: stimulus relevancy (attending vs. not attending 
a given field), eccentricity ( $10 \mathrm{deg}$ vs. $30 \mathrm{deg}$ ), visual field in which the evoking stimulus was presented (right vs. left), lobe from which VEPs were obtained (right vs. left), and primacy of the lobe from which VEPs were obtained (direct stimulation via projections from hemiretina activated vs. indirect stimulation via the corpus callosum). These will be abbreviated hereafter as the relevancy, eccentricity, visual-field, lobe, and primacy factors. Each subject participated in a $2 \mathrm{~h}$ session consisting of eight 5 -min trials, the last four being counterbalanced replications of the first four. A 15-min break separated the first set of four trials from the second set, all other intertrial intervals being $5 \mathrm{~min}$. In each trial, VEPs were obtained from each lobe to stimuli presented concomitantly but never simultaneously in each visual field at one of the two eccentricities; one field was relevant, and the other was irrelevant.

In Experiment 2 the design consisted of four factors having four levels with respect to relevancy and two levels each with respect to lobe, visual field, and primacy. VEPs were obtained from both lobes during each condition. Relevancy was counterbalanced across the two fields for the focused attention condition.

\section{Subjects}

Twenty-four undergraduate students participated, 12 in each experiment. All had normal or corrected vision. All were naive with respect to the purpose of the study. None had previously participated in a VEP study. Most were in their early 20s; none was over 40 . Most were female.

\section{Apparatus and Procedure.}

Experiment 1. Subjects discriminated whether a given stimulus presentation to the relevant visual field consisted of a single flash or of randomly interspersed double flashes occurring in quick succession, and they reported at the end of the run the total number of double flashes seen. The doublets, which occurred about $20 \%$ of the time, were separated by $100 \mathrm{msec}$, well beyond each subject's temporal discrimination threshold for the stimulus parameters used. VEPs were recorded to single flashes only, and there were 100 flashes for each visual field in a given trial.

Stimulus flashes subtending 1 deg of visual angle (VA) were delivered by fiber optics light guides independently illuminated by Grass PS-2 photostimulators. Using solid state modular equipment, stimuli were presented randomly in each visual field. The interstimulus interval varied unpredictably from $.5 \mathrm{sec}$ to $5 \mathrm{sec}$, the average being about $2 \mathrm{sec}$. The subject could not predict above chance whether the next stimulus would appear in the relevant or the irrelevant field or, with any accuracy, when the next stimulus would appear in either field. Stimulus flashes were approximately 2 log units above a background luminance level of $1 \mathrm{~mL}$.

Occipital VEPs were detected with electrodes placed at $\mathrm{O}_{1}$ and $\mathrm{O}_{2}$, amplified with Grass EEG equipment, and signal averaged.

To check for eye movement artifacts, a continuous electrooculogram (EOG) was obtained during each trial with electrodes placed at the external canthi of each eye. A Grass dc unit magnified the signal so as to produce approximately a $10-\mathrm{mm}$ pen deflection for a 20-deg saccade. Eye shifts as small as $1 \mathrm{deg}$ were detectable. EOGs were monitored throughout each run. Since pen drift usually occurs in dc recordings, a procedure was adopted to determine whether such drift was due to eye movements. This entailed instructing the subject immediately at the end of each trial to fixate the target. No movement signified the subject was properly fixating and that any pen drift was due to causes other than slow eye drift. To check for the possibility that minute, reflexive eye movements might be time locked to the stimulus flashes, averaged EOGs were obtained in a preliminary run for each subject to 100 flashes presented in each visual field (one relevant, the other irrelevant). Anyone showing such movement in excess of $1 \mathrm{deg}$ was to be excluded from the study.
Counterbalancing procedures controlled for order effects, differences in the brightness of the two stimulus delivery units, and differences in the amplification factor of the two channels used to record VEPs.

Experiment 2. The same basic controls and recording procedures were employed as were used in Experiment 1. Under the both-fields-irrelevant condition, a procedure not previously used required that subjects perform a foveal discrimination task. This entailed counting the number of times the fixation stimulus, a dim red light subtending about $30 \mathrm{~min}$ VA, varied in brightness during the course of a trial. The rate of change varied irregularly from 3 changes/sec to 1 change $/ 3 \mathrm{sec}$, with an average rate of approximately $1.5 \mathrm{change} / \mathrm{sec}$. The brightness variations of approximately $1 \mathrm{log}$ unit were well above threshold and occurred independently of the peripheral stimulus flashes that triggered the averaging computer.

\section{RESULTS}

\section{Eye Movement Artifacts}

Examination of the EOG records revealed that none of the subjects made shifts from the fixation target in excess of $1 \mathrm{deg}$ during a trial. Also, the instruction to "fixate the target" immediately at the end of each run resulted in no pen deflection greater than $1 \mathrm{deg}$ for anyone. It may be concluded, therefore, that the subjects did maintain proper fixation within the limits of physiological nystagmus.

Averaged eye movements obtained in the preexperimental run revealed that 23 of 24 subjects gave no noticeable indication of stimulus-locked reflexive eye movements. The average magnitude of deflections was less than $15 \mathrm{~min}$ VA for all subjects except one, well within the range of normal physiological nystagmus. One subject showed a tendency to shift the eyes slightly (approximately $30 \mathrm{~min}$ VA), but always in the same direction regardless of the visual field in which the stimulus appeared. Given the visual field counterbalancing procedures used and the minuteness of the movement, this subject was allowed to participate in the experiment.

\section{Behavioral Data (Counting Accuracy)}

In Experiment 1, the median error in counting double flashes was $8.5 \%$. The error was less than $10 \%$ for six subjects, between $10 \%$ and $20 \%$ for four, and between $20 \%$ and $50 \%$ for two. The errors tended to be overcounts: 8 of the 12 counted more doublets than were actually presented.

In Experiment 2, the median error depended on whether the subjects counted doublets under the focused or divided attention condition. When counting in only one field, the error was $9.8 \%$, about the same as in Experiment 1 . The error distribution also was similar, with 8 of the 12 subjects counting more than actually were presented. When counting doublets in both fields, the error was eight times greater than that for one field only, the median being $80.6 \%$. The error was over $100 \%$ for five subjects, $70 \%-80 \%$ for two, $20 \%-30 \%$ for one, and less than $10 \%$ for four. All subjects, except two, made overcounting errors; those who undercounted 
barely did so, the median errors being $-2.4 \%$ and $-5.6 \%$.

\section{Averaged VEPs}

VEP-component identification. Averaged VEPs are exemplified in Figure 1. Four sets of 24 superimposed tracings are depicted, 2 from each of 12 subjects. The tracings on the left were obtained from the relevant field; those on the right were obtained from the same field when irrelevant. Top tracings are right-lobe responses; lower ones are left-lobe responses. The major deflections or "components" in the white (groupaverage) tracings may be identified by polarity $(\mathrm{P}$ or $\mathrm{N})$ and average latency (onset and peak): (1) $\mathrm{B}$ (baseline) ${ }_{70}$ $\mathrm{P}_{120}$, (2) $\mathrm{P}_{120}-\mathrm{N}_{170}$, (3) $\mathrm{N}_{170}-\mathrm{P}_{230}$, (4) $\mathrm{P}_{230}-\mathrm{N}_{280}$, and (5) $\mathrm{N}_{280}-\mathrm{P}_{330}$. These will be referred to hereafter as components (C) 1, 2, 3, 4, and 5. Individual VEPs were quantified by measuring the vertical distance between the onset and offset of each component, with the constraint that the latencies used for a given subject had to be within $10 \mathrm{msec}$ of the group-average latencies specified above.

Attention effects. The group-averaged VEPs obtained in Experiment 1 for each experimental condition are shown in Figure 2. The tracings are categorized with respect to visual field, relevancy, lobe, primacy, and eccentricity. Although significant differences were observed with respect to each of these variables, only those results pertaining to relevancy are reported here. ANOVAs performed on each of the five VEP components revealed in every case significantly greater amplitudes for the relevant than for the irrelevant field $(p<.01)$. A comparison of the pairs of tracings in each row of Figure 2 reveals these differences in most instances. Of special interest is the fact that $\mathrm{C} 1$ was

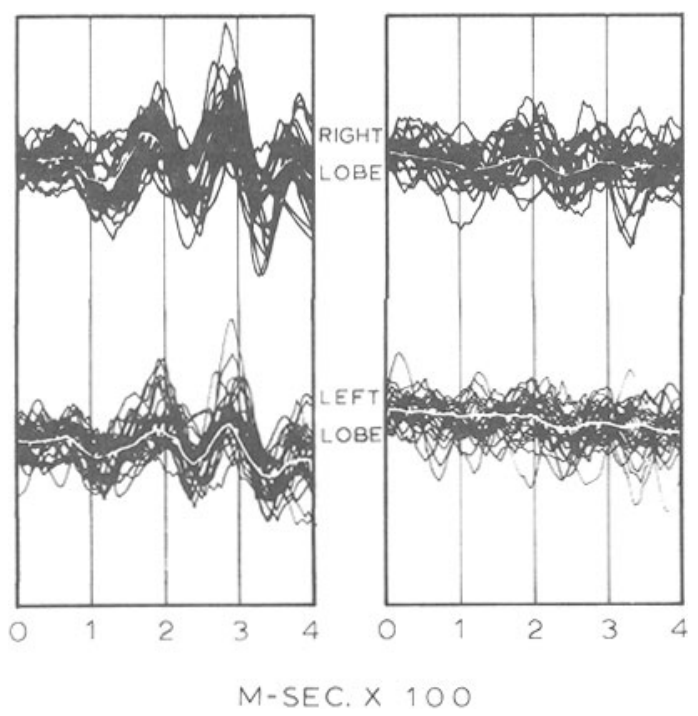

Figure 1. Superimposed tracings of VEPs obtained from 12 subjects (two per subject) in Experiment 2.

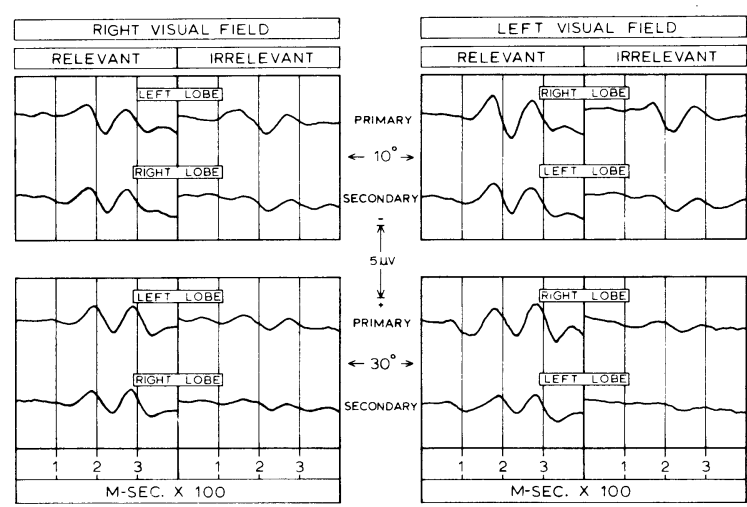

Figure 2. Group-averaged VEPs obtained with respect to relevancy, visual field, lobe, and primacy in Experiment 1 (see text).

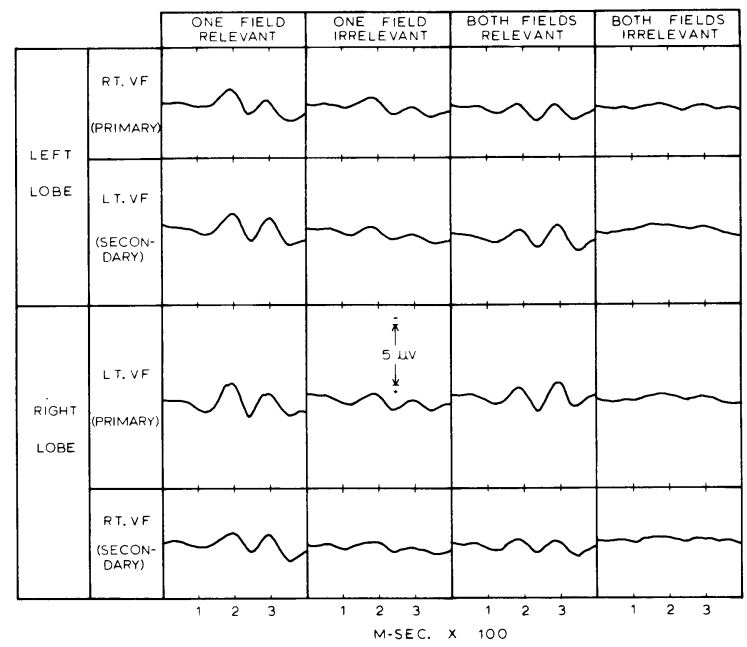

Figure 3. Group-averaged VEPs obtained with respect to relevancy, visual field, lobe, and primacy in Experiment 2 (see text).

greater for the relevant field. Also of interest was the fact that C3, C4, and C5 showed greater differences with respect to relevancy at the 30-deg than at the 10-deg peripheral location $(\mathrm{p}<.01$ in two instances and $\mathrm{p}<.05$ in the other).

Results of Experiment 2 are depicted in Figure 3, wherein group-averaged VEPs are categorized by relevancy, lobe, visual field, and primacy. ANOVAs again revealed that all five deflections were affected by relevancy ( $\mathrm{p}<.01$ in every case). Between-column comparisons in Figure 3 reveal this effect, VEPs being largest when only one field was relevant, next largest when both were relevant, third largest when one field was irrelevant, and smallest when neither field was relevant. The extent of the relevancy effect depended on which field was stimulated and which lobe was recorded from $(\mathrm{p}<.05$ for $\mathrm{C} 1, \mathrm{C} 2$, and $\mathrm{C} 4$; the effect was greater for the left field and right lobe than for the right field and left lobe. 


\section{DISCUSSION}

\section{Attention Effects}

Experiment 1. Assuming $\mathrm{C} 1$ arises from the striate cortex, as animal data suggest (Creutzfeldt \& Kuhnt, 1973), the results support the precortical filtering hypothesis. The results could be due to facilitation of relevant information transmission, inhibition of irrelevant transmission, or both.

The effect of relevancy on C2-C5 is consistent with the results of the Eason et al. (1969) study. Van Voorhis and Hillyard (1977) were unable to modulate a component labeled $\mathrm{N}_{1}$ (peak latency of $155 \mathrm{msec}$ ), which appears analogous to $\mathrm{C} 2$ $\left(P_{120}-N_{170}\right)$ of the present study. The discrepancy may be due to differences in quantification; peak-to-trough measures were used in this and the Eason et al. study, whereas baseline-to-peak (or trough) measures were used in the Van Voorhis and Hillyard study.

The greater effect of relevancy on certain VEP components at 30 -deg than at 10 -deg eccentricity suggests filtering is more "efficient" the farther apart the relevant and irrelevant stimuli are in physical space. Efficiency may depend in part on the degree to which relevant and irrelevant stimuli share the same sensory channels: the less overlap, the greater the efficiency.

Experiment 2. The effects of focused attention were consistent with those observed in Experiment 1 and thus constitute further corroboration of the Eason et al. (1969) study.

In the divided attention condition, the reduced amplitude of the VEPs obtained when both fields were relevant, compared with one being relevant, suggests that each was attended approximately half the time. Although an increase in counting error due to such division of attention might be expected, an eight-fold increase seems excessive and needs explanation. Except for $\mathrm{C} 2$ discussed above, the divided attention data generally are consistent with the results obtained by Van Voorhis and Hillyard (1977) for the visual system and by Parasuraman (1978) for the auditory system. The extreme attenuation of the VEP under the both-fields-irrelevant condition appears due to the demanding foveal task the subjects had to perform, since passively fixating a foveal stimulus does not further reduce the amplitude of the VEP from that obtained during focused peripheral attention (Van Voorhis \& Hillyard, 1977).

\section{Artifacts and Controls}

The procedures followed and results obtained in the present study should satisfactorily answer criticisms of the Eason et al. (1969) study. Conscientious individuals, including naive subjects, can and do maintain appropriate fixation when instructed to do so. Most of the subjects in the Eason et al. study were experienced observers. Also, the corroborative results obtained, using completely randomized procedures for presenting stimuli in the two visual fields, indicate that the findings of the Eason et al. study were not biased by differential activation effects resulting from quasirandom stimulus presentation procedures (Näätänen, 1975).

\section{REFERENCES}

Creutzfendt, O. D., \& Kuhnt, U. Electrophysiology and topographical distribution of visual evoked potentials in animals. In R. Jung (Ed.), Handbook of sensory physiology. Berlin: Springer-Verlag, 1973.

EAson, R. G., HARTer, M. R., \& White, C. T. Effects of attention and arousal on visually evoked cortical potentials and reaction time in man. Physiology \& Behavior, 1969, 4, 283-289.

Hasselt, van P. The centrifugal control of retinal function: A review. Ophthalmological Research, 1973, 4, 298-320.

Hillyard, S. A., Picton, T. W., \& Regan, D. Sensation, perception, and attention: Analysis using ERPs. In E. Callaway, P. Tueting, \& S. H. Koslow (Eds.), Event-related brain potentials in man. New York: Academic Press, 1978.

Livingston, R. B. Sensory processing, perception, and behavior. New York: Raven Press, 1978.

NÄ̈̈ÄNEN, R. Selective attention and evoked potentials in humans-A critical review. Biological Psychology, 1975, 2, 237-307.

OAtman, L. C., \& Anderson, B. W. Suppression of the auditory frequency following response during visual attention. Electroencephalography and Clinical Neurophysiology, 1980, 49, 314-322.

Parasuraman, R. Auditory evoked potentials and divided attention. Psychophysiology, 1978, 15, 460-465.

VAN Voorhis, S., \& Hillyard, S. Visual potentials and selective attention to points in space. Perception \& Psychophysics, 1977, 22, 54-62. 